\title{
"Slippage" in the talent development environment of elite junior tennis players
}

\section{Callum Gowling}

United Kingdom.

\begin{abstract}
"Slippage" occurs when there is a mismatch between the intended message communicated (by coach, parent, or talent development environment) and the received message by junior tennis players. The concept of slippage has received attention in educational settings, but research has yet to investigate the possible effects on junior tennis players. The purpose of this study was to examine the experiences of $8 \mathrm{UK}$, elite junior tennis players and describe what it is like to perform in the elite junior context. An Interpretative Phenomenological Analysis (IPA) of 8 elite junior tennis players (playing LTA Grade 2 and upwards) shows their insights into elite junior tennis. This study found that (a) participants seek meaning behind their tennis experiences and they infer messages from their environment that are not intended, (b) "slippage" is associated with unintentional pressure, and (c) "slippage" is associated with a reduction of junior tennis players' confidence. The findings of this research contribute to an evolving, problematic epistemology of sports coaching and confirms that coaches can lose control of their intended message as players infer their own meanings from interactions within tennis. The findings present governing bodies opportunities to inform coach education literature and consider how the difference between the intended coaching message and the received coaching message can impact the emotional state of junior tennis players.
\end{abstract}

\begin{abstract}
Key words: interpretative phenomenological analysis, elite junior tennis, slippage
\end{abstract}

Received: 10 April 2021

Accepted: 19 May 2021

Corresponding author: Callum Gowling. Email: callumgowling@ btinternet.com

\section{INTRODUCTION}

The talent development environment (TDE) has a profound physical and emotional effect on the athletes' who train within such environments (Henriksen et al., 2010). Youth performance coaches create intensive preparation programmes to enable young people to train in specialised environments with desirable values for sporting achievement. TDE's contain beliefs, values, attitudes, dispositions, and subtle intuitions acted out via interactions between players, coaches, and parents in the elite junior tennis environment.

Without careful monitoring and criticality of the conscious or sub-conscious messages that children see and hear, it is possible that flawed learning outcomes are absorbed. "Slippage" occurs when there is a difference between the intended message (from coach, parent, or the talent development environment) and the received message by the player (Cassidy \& Tinnings, 2004). Despite governing body's best intentions, seemingly well-structured training programmes are associated with players who experience unintentional pressure and low levels of confidence due to misinterpretation of messages in the TDE. For example, players repeatedly told about areas to improve so they can reach higher levels, can interpret this as not being good enough and reduce confidence.

A deeper understanding of 'slippage' and the real world impact on the emotional state of elite junior tennis players would help junior tennis coaching through better provision of TDE's that

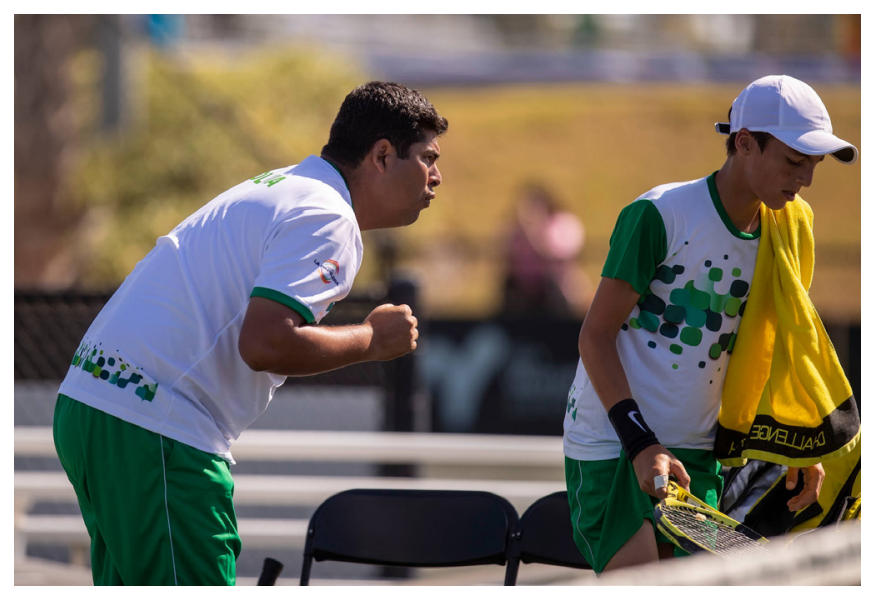

are more considerate of social learning. It would be helpful to understand what it is like for junior tennis players to live, eat, and breath a sport known to; prioritise ranking / selection / representation (Lauer et al., 2010); include a prominent performance narrative (Douglas \& Carless, 2012); rely on financial investment from parents (Dunn et al., 2016); contain high levels of interpersonal conflict (Wolfenden \& Holt, 2005; Smoll, Cumming, \& Smith, 2011, Gowling, 2019).

Elite junior tennis "a multifaceted social setting involving complex relationships between players, parents, and coaches" (Wolfenden \& Holt, 2005, p. 122). The specific nature of the social context in which talent development in tennis occurs 
contributes to the reality of junior tennis players. For example, tennis players commit to tennis earlier than in other sports, and early specialisation means coaches, players, and parents interact with each other intensely for many years (Knight \& Holt, 2014). The intensity and frequency of coach, player, and parent interactions will inevitably affect the children involved and this deserves more attention in research.

This article looks at the experiences of $8 \mathrm{UK}$ elite junior tennis players and illustrates they are sensitive to more than simple words and instructions given directly to them during training. The responses show participants searching for their own meaning relating to their tennis experiences and they infer messages from their surroundings that are not intended.

\section{METHOD}

This study was an interpretative phenomenological analysis (IPA) of 8 elite junior tennis players in the UK. The participants included 4 males and 4 females aged between 11 and 18 years old. The participants trained in the following areas: Northern England (4), Southern England (3), and Wales (1). Participants were Junior elite, and this was defined as competing at national level competition and above (Rees et al., 2016). Interviews were semi-structured, and the aim was to understand what it was like for participants to play elite junior tennis. Interviews lasted between 45 minutes and 90 minutes and were audio recorded. Interview transcripts were transcribed verbatim, printed out, and analysed following the IPA procedure.

\section{RESULTS}

The results show (a) junior tennis players seek meaning behind their tennis experiences and they infer messages from their environment that are not intended, (b) "slippage" can be associated with unintentional pressure, and (c) "slippage" can be associated with reduction in confidence.

Junior tennis players seek meaning behind their tennis experiences and they infer messages from their environment that are not intended.

Junior tennis players are not non-thinking pawns, who blindly attend training and competition, oblivious to the sacrifices, behaviours, judgements, and expectations of others. Often by the time players reach yellow ball (U12) they have experienced many years of training and competition. Years of interactions in tennis inform players interpretations about the sacrifices people make for their tennis, leading to questions about whether there is more to tennis than fun. Amy described her views on tennis culture: "Everyone says, relax and play your game. Someone should tell the adults to relax. Why are they all so frantic"? Due to the amount of training, travel, and competition involved in the elite junior context, it is natural that coaches and parents make substantial time, financial, and emotional commitments to help players and give them opportunities to succeed. However, sometimes the message received by the player can 'slip' to one of single minded dedication to tennis to the detriment of other areas of life. Sam described how tennis became a dominant source of conversation: "My dad only really talks to me about tennis these days. It's like that's all that matters". Understandably, parents want their children to know they are interested in their development. However, the support network around players is unable to fully attend to social learning and the messages players derive from daily interactions. Tomas illustrated how his parents concern for his development could be perceived as a player stressor:

When I've lost, I sit at the top of the stairs at home and listen to my mum and dad talking about my tennis. I know the next day we will have a family discussion about my programme and where I'm going in tennis. It becomes this huge issue.

Scrutinising daily interactions and attributing 'meaning' to such interactions was fundamental to the participants perceiving tennis as taking on increasing importance in the lives of people in their environment. Parent - coach interactions contributed to players feeling tennis was a priority for everybody involved in their development. James said, "I reckon my dad speaks to my coach more than my mum these days. They are on the phone for hours". It is important that parents and coaches work together to benefit junior players, but James went on to describe a detrimental effect on his tennis participation, "I feel guilty sometimes when I lose. Everyone puts so much effort into my tennis. I just know everyone is gutted when I lose". The participants consistently described situations where they searched for meaning behind the actions of people in their development, often making their own interpretations that informed their attitude towards tennis and what it means to them.

\section{"Slippage" can be associated with players feeling unintentional pressure}

A consistent theme throughout the responses highlighted that junior players were intrigued by 'why' the behaviours they witnessed around tennis occurred. For example, Oli said "I don't get why my coach reacts like he does. If I lose, it is always followed by 'you've got to work harder'. I'm like, I am working hard". Once players inferred their own meaning, there was often contradiction between the spoken words they received (e.g. relax, enjoy yourself, have fun) and the perceived behaviours they witnessed from coaches, parents, other players (anxiety, upset, anger, disappointment). Sarah said "You start to dread tournaments. You don't want to let anyone down. It would be nice to play freely but tournaments are tense. Everyone is so uptight". Junior tennis players have a preoccupation with pleasing parents and coaches through their performances because they are aware of the sacrifices made (Lauer et al., 2010). Wanting to please others associated with their development contributes to the pressure that players feel during competition.

The participants continued to describe examples of unintentional pressure. Participants seemingly interpreted much of their post tournament feedback as 'criticism' rather than constructive feedback. A mismatch between the intended coaching message (i.e. work hard to improve) and the received message (i.e. not good enough) seemed to affect players enjoyment of competition. Amy said "I hate it when my coach comes to watch. All the pressure and the criticism is hard to take". Rachel described a similar experience "I know they are there to support (parent/coach) but you see their reaction when you miss. I'm not missing on purpose". James concurred with Amy and Rachel, "When my coach is there, I'm scared to hit my backhand down the line. It's my best shot, but if I play it, I get told off". Lorimer and Jowett (2009) highlight the importance of empathetic accuracy in coach, athlete, parent relationships. The evidence suggests that some junior tennis players may be inaccurate in how they assess coach / parent expectations, resulting in players experiencing pressure. 


\section{"Slippage" can be associated with reduction in confidence}

Participants consistently questioned their daily interactions, inferred their own meanings (slippage), and described unintentional pressure due to wanting to please their coaches and parents. Unsurprisingly, there was evidence that players experienced issues with self-confidence. Sam said "I'd rather play ITF's. When you play in this country, it can be embarrassing because you know everyone is talking about you when you lose". There was agreement throughout the responses that it was difficult to play freely, because the participants imagined negative judgements by those within tennis. Rachel described discomfort with competition "You feel like if you're top seed, people want you to lose. I hate being number 1 seed". A natural consequence of performing well is players carry high rankings. Unfortunately, some players interpret their high ranking as added pressure which made it difficult to play freely. Tomas added, "I'd much rather be the underdog. When you are number 1 or 2 seed you know you'll play bad". When I asked Tomas why he knew he would play bad, he said "everyone expects you to win, but wants you to lose". Junior tennis is a competitive environment which includes interpersonal conflict (Gowling, 2019) and participants were aware of this. There is evidence to suggest that players assumed the competitiveness they witnessed amongst player, coaches, and parents created an unhealthy atmosphere that impacted on their perceived ability to perform under pressure.

\section{DISCUSSION}

The data in this study highlights several ways that junior tennis players are affected by their talent development environment:

First, the findings of this study add to the work of Lorimer and Jowett (2009). Empathetic accuracy between player, coach, and parent aids the healthy development of junior tennis players. Failure to maintain an accurate understanding of the intended message behind tennis behaviours can cause the intended coaching message to 'slip' and potentially damage the players experience in tennis (Cassidy \& Tinnings, 2004)

Second, the findings confirm our understanding that unintentional pressure exists in elite junior tennis (Lauer et al., 2010). Players understand the sacrifices their parents make to provide opportunities in tennis. Without regular communication and criticality of the talent development environment it is possible the intended message of care, provision of opportunity, and self-improvement can slip to a received message of pressure to justify sacrifice.
Finally, the findings confirm that elite junior tennis is a competitive environment that places a high cognitive load on young people (Wolfenden \& Holt, 2005; Gowling, 2019). Junior tennis players require a cohesive support network to help them keep perspective on their tennis experiences in light of contradictory messages they infer from the talent development environment (e.g. competition, and criticism)

\section{CONCLUSION}

The findings of this research contribute to an evolving, problematic epistemology of sports coaching and confirms that coaches can lose control of desirable coaching messages as players infer their own meanings from interactions within tennis. The findings present governing bodies opportunities to inform coach education literature, and player support systems due to differences between the intended message (from coach, parent, or environment) and the received message that impacts on the emotional state of junior tennis players.

\section{REFERENCES}

Cassidy, T. \& Tinning, T. (2004). "Slippage" is not a dirty word: considering the usefulness of Giddens' notion of knowledgeability in understanding the possibilities for teacher education, Teaching Education, 15:2, 175-188, DOI: 10.1080/1047621042000213601

Douglas, K. \& Carless, D., (2012). Stories of success: Cultural narratives and personal stories of elite and professional athletes, Reflective Practice: International and Multidisciplinary Perspectives, 13:3, 387-398, DOI: $0.1080 / 14623943.2012 .657793$.

Dunn, C., Dorsch, T., King, M., \& Rothlisberger, K. (2016). The impact of family financial investment on perceived parent pressure and child enjoyment and commitment in organized youth sport. Family Relations, 65, 287-299. https://doi.org/10. 1111/fare.12193.

Gowling, C. (2019). Understanding the pressures of coaching: In sights of young UK coaches working with elite junior tennis players. ITF Coaching \& Sport Science Review, Vol 79, pp 19 - 21.

Henriksen, K., Stambulova, N., Kaya Roessler, K., (2010) Holistic approach to athletic talent development environments: A successful sailing milieu. Psychology of Sport and Exercise, Vol 11.

Knight, C., \& Holt, N., (2014). Parenting in youth tennis: Understanding and enhancing children's experiences. Psychology of Sport and Exercise, 15, 155-164.

Lauer, L. Gould, D. Roman, N. \& Pierce, M. (2010), Parental behaviours that affect junior tennis player development, Psychology of Sport and Exercise, Vol 11, p 487 - 496

Lorimer, R. \& Jowett, S. (2009) Empathic Accuracy, Meta-Perspective, and Satisfaction in the Coach-Athlete Relationship, Journal of Applied Sport Psychology, 21:2, 201-212. DOI: 10.1080/10413200902777289.

Rees, T. Hardy, L. Gullich, A. Abernethy, B. Cote, J. Woodman, T. Montgomery, H. Laing, S. Warr, C. (2016). The Great British Medalists Project: A Review of Current Knowledge on the Development of the World's Best Sporting Talent. Sports Med, Vol 46, pp 1041 - 1058.

Smoll, F. L. Cumming, S. P., and Smith, R. E., (2011). Enhancing Coach Parent Relationships in Youth Sports: Increasing Harmony and Minimising Hassle, International Journal of Sports Science and Coaching, vol 6: 1.

Wolfenden, L. E. \& Holt, N. L. (2005) Talent Development in Elite Junior Tennis: Perceptions of Players, Parents, and Coaches, JOURNAL OF APPLIED SPORT PSYCHOLOGY, 17:2, 108-126, DOI: $10.1080 / 10413200590932416$.

\section{Copyright $\odot 2021$ Callum Gowling

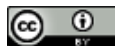 \\ This text is under a Creative Commons BY 4.0 license}

You are free to Share - copy and redistribute the material in any medium or format - and Adapt the content - remix, transform, and build upon the material for any purpose, even commercially under the following terms:

Attribution: You must give appropriate credit, provide a link to the license, and indicate if changes were made. You may do so in any reasonable manner, but not in any way that suggests the licensor endorses you or your use. 